\title{
Quality assurance for synovial fluid examination for crystals: an improved method
}

\author{
Neil W McGill, Vicki G McGill
}

\begin{abstract}
Objective-To determine the best method of preparing synovial fluid specimens for use in quality assurance (QA) surveys designed to assess accuracy of crystal identification.
\end{abstract}

Methods-A previously published method (A) was compared with a new method (B) in the setting of a QA survey. Ten Australian, one New Zealand, and one Hong Kong hospital laboratories took part in the survey. Each laboratory examined six different synovial fluid specimens prepared using method $A$ (first round) and a separate six specimens using method $B$ (second round). In method $A$, a drop of synovial fluid on a glass slide was surrounded by a rim of Ultramount, sealed with a coverslip, and distributed. The participating laboratory did not need to perform any processing of the specimen before examination. In method $B$, a capillary tip was filled with synovial fluid, heat sealed, and distributed. The fluid was expelled onto a glass slide in preparation for examination after arrival in the participating laboratory.

Results-Using method A 36 of 71 (51\%) of the specimens were rated as satisfactory, compared with 53 of $61(87 \%)$ of the specimens using method B (Fisher's exact test, $\mathbf{p}<0.001$ ).

Conclusions-An improved method of preparation of synovial fluid specimens for QA surveys is described. Using the new method it is feasible to perform a synovial fluid QA survey covering a large area (Australasia).

(Ann Rheum Dis 1997;56:504-506)

Synovial fluid examination for crystals is a crucial step in reaching an accurate diagnosis of crystal induced arthritis. Its importance is firmly established and the result strongly influences treatment, which, in the case of gout, frequently entails lifelong drug treatment. A false result could result in inappropriate treatment for many years and thus, accuracy is vital. Several studies, involving laboratories in the USA, ${ }^{12}$ Finland,${ }^{3}$ and Australia, ${ }^{4}$ have shown that the reliability of synovial fluid examination, even in university and teaching hospitals, is imperfect both with respect to a failure to detect crystals (sensitivities of $78 \%{ }^{2}$ $79 \%{ }^{4}$ for urate, and $12 \%,{ }^{2} 67 \%{ }^{4}$ for calcium pyrophosphate dihydrate (CPPD) and false positive results $\left(16 \%,{ }^{2} 24 \%,{ }^{3} 11 \%{ }^{4}\right)$. The desirability of an ongoing quality assurance
(QA) programme for synovial fluid analysis has been recognised but has not occurred because of logistical difficulties in providing synovial fluid samples to laboratories, particularly over large distances.

Ideally, the QA programme should incorporate both preparation and examination of synovial fluid specimens. Studies using comparatively small numbers of laboratories within close proximity ${ }^{14}$ have used $0.5-0.75 \mathrm{ml}$ aliquots of each specimen but this is not feasible for large numbers of laboratories (the volume originally aspirated from the joint is usually $<5 \mathrm{ml}$, enough for only 6-10 laboratories). Preparation of slides before distribution allows the use of much smaller amounts of fluid but, despite efforts to reduce dehydration of the synovial fluid after slide preparation, ${ }^{5}$ this method is limited by deterioration of the specimen between preparation and examination. We have performed a series of pilot QA surveys of synovial fluid analysis for crystals involving laboratories in Australia, New Zealand, and Hong Kong. In the initial studies, many laboratories indicated that the appearance of the synovial fluid samples differed from fresh specimens and that artefacts were common. In response to the feedback received, we have modified the preparation method in an attempt to deliver good quality specimens to the laboratories in a manner that will permit an ongoing QA programme.

\section{Methods}

SAMPLE COLLECTION AND STORAGE

Synovial fluid specimens, which had been collected into sterile plain or non-crystalline lithium heparinised containers, were stored in plastic tubes at $-70^{\circ} \mathrm{C}$. This storage method has been previously shown to preserve synovial fluid crystal numbers and cellular morphology for many weeks ${ }^{6}$ although any small loss of crystals that may have occurred would have been of no consequence as the reference findings were obtained after thawing. On the day of preparation, the fluids were thawed, examined for crystals, and then processed for the QA programme.

SELECTION OF LABORATORIES

An invitation to participate in the pilot programme was sent to laboratories already using the Royal College of Pathologists of Australasia (RCPA) microbiology QA programme. One hundred and sixty eight laboratories expressed a wish to participate. Because this was well in excess of the number required, a sample was chosen to cover laboratories over a range of distances from the source, and to 
include both teaching hospital/reference and smaller laboratories. The hospitals that participated were: St Vincents, Westmead, Prince of Wales, Rachel Forster (all NSW), Fairfield (Victoria), Institute of Medical and Veterinary Science (SA), Townsville General (QLD), Royal Hobart (Tasmania), Royal Darwin (NT), King Edward Memorial (WA), Wellington Public (New Zealand), and Sai Ying Pun JC Polyclinic (Hong Kong).

PREPARATION OF SPECIMENS

After thawing at room temperature, synovial fluid specimens were hand shaken, examined with polarised microscopy, and then prepared. Only fluids containing sufficient crystals to ensure that most high powered microscope fields $(400 \times)$ contained crystals were used. Two different methods of preparation were used and compared.

(A) Initial method, as described by Schumacher5: a drop of synovial fluid was placed on a clean glass slide, surrounded by a rim of Ultramount, and sealed with a coverslip. The prepared slides were placed in protective cardboard containers while the Ultramount dried.

The following day the slides were examined (any slide, where the sealing process appeared less than ideal, was discarded) and then distributed in sealed, insulated (but not chilled) packages via Australia Post. The distribution was carried out by RCPA Quality Assurance Programs Pty Ltd (Royal North Shore Hospital, Sydney) in accordance with IATA dangerous goods regulations.

Upon arrival at the laboratory the slides could be examined without the need for any processing.

(B) New method, devised by ourselves: using a manual pipette, $25 \mu \mathrm{l}$ capillary tips (Corbett Research, CT-960) were filled with synovial fluid, heat sealed, and labelled. The following day they were distributed as above.

Upon arrival at the laboratory, the capillary tip needed to be sliced off with a scalpel and the plunger depressed to expel the fluid onto a slide for examination.

\section{RATING OF QUALITY OF SYNOVIAL FLUID}

PREPARATIONS

Laboratories were asked to rate the quality of the specimens (as determined at the time of microscopic examination) as satisfactory or poor. In light of the pilot nature of the study, it was felt best to use a highly qualitative grading method. Comments were encouraged. The rating procedure occurred twice - that is, at the time of each survey, once for method A and once for method B. In addition, on the response sheet for method $\mathrm{B}$, participants were asked whether method $\mathrm{B}$ provided superior or inferior results to method $\mathrm{A}$.

EXAMINATION FOR CRYSTALS

The participants were asked to examine the slides (method A) or to prepare slides using the fluid contained in the capillary tips (method B), and to identify which fluids contained crystals and the nature of the crystals. The response sheet allowed participants to nominate the presence of crystals as: urate, CPPD, none, or other (to be specified if possible by the respondent), and also allowed comments to be made.

\section{Results}

QUALITY OF SYNOVIAL FLUID PREPARATION COMPARING METHODS A AND B

Each laboratory was asked to comment on the quality of six different slides (A) or fluids (B) in each survey, thus there were a total 72 samples in each survey. Some laboratories failed to score every specimen. In the initial survey, in which synovial fluid specimens were sealed on glass slides before distribution (method A), 36 of $71(51 \%)$ of the slides were rated by the participating laboratories as satisfactory. In contrast, the survey using method B, 53 of 61 $(87 \%)$ of the specimens were described as satisfactory. Of the 12 laboratories taking part in this study, 11 indicated that method B provided better quality specimens than method A.

The exact meaning of 'satisfactory' was not defined but those deemed 'unsatisfactory' by the laboratories were described as being very unlike fresh synovial fluid samples in appearance, usually because of drying artefact in method A and clumping/gelling of the fluid in method $\mathrm{B}$.

As a different set of synovial fluids was used in each survey, it is possible that the quality of the samples before preparation was different. However, the pronounced difference in preference for method B is unlikely to result from a quality difference of the synovial fluids used.

EXAMINATION FOR CRYSTALS

There was no 'gold standard' for the presence or absence of crystals and thus the reference findings (obtained by one of the authors, $\mathrm{N}$ $\mathrm{McG}$ ) were used as a substitute. The reference findings were obtained immediately before preparation of the samples prior to distribution, that is after thawing and shaking. As a different set of synovial fluids was used in each survey, it is possible that the ease of identification of crystals in the samples was different and thus direct comparison of the crystal identification results between the two surveys should not be made.

In both surveys (abbreviated below as $\mathrm{A}$ and B) six synovial fluids samples were sent to each of the 12 participating laboratories (total of 72 samples). On both occasions two of the fluids contained urate, two contained CPPD, and two contained no crystal visible with light microscopy. Thus, in total, there were 24 'urate', 24 'CPPD', and 24 'no crystal' samples in each survey.

Of the 24 urate samples, 24 (in survey A) and 18 (in survey $\mathrm{B}$ ) were identified as containing urate crystals. Of the 24 CPPD samples, 12 (A) and 16 (B) were identified as containing CPPD crystals. Of the 24 'no crystal' samples, false positive findings were reported in four (A) and two (B). Of the 48 urate and CPPD samples, false positive crystal 
identifications (for example, urate reported in a CPPD sample) were reported in two (A) and four (B).

There was considerable variability in the reliability of different laboratories. The total correct scores (maximum 12) for the laboratories were $12,11,10,10,10,9,9,9,8,7,7,5$.

STATISTICAL ANALYSIS

Fisher's exact test was used. Because there were some missing data (some laboratories failed to indicate 'satisfactory' or 'poor' when rating the quality of some specimens) significance was calculated first ignoring the missing data $(\mathrm{p}<0.0001)$, and second using a 'worst case' scenario, assuming the missing data would have all worked to increase similarity between the two groups $(p<0.001)$

\section{Discussion}

Reliable synovial fluid examination for crystals plays a vital part in the diagnosis of gout and CPPD deposition disease. Previous surveys have shown comparativley high rates of error even in teaching hospital and reference laboratories. ${ }^{1-4}$ Most laboratory tests are now monitored by QA programmes but synovial fluid examination has not been incorporated in these programmes. Difficulties with supply of suitable samples and the lack of a satisfactory method of distributing samples over large distances or to large numbers of laboratories probably account for the lack of routine synovial fluid examination QA programmes.

Drying artefacts in the synovial fluid samples severely limit the usefulness of the previously described method $^{5}$ for preparing samples for distribution. The new method described here overcomes some of the problems associated with preparation of synovial fluid samples. The advantages include: (1) small sample size allowing a large number of laboratories to examine fluid from the original sample enabling valid comparisons to be made between laboratories, (2) avoidance of drying artefact, (3) requirement for the participating laboratory to prepare the slide and coverslip (allowing problems related to dusty slides, etc, to be detected), (4) preparation of micro pipettes is faster, requires less skill, and essentially eliminates failures compared with preparation of slides using the previously published method. Despite these benefits, some problems still exist: viscous fluids tend to clump and can be difficult to detach from the pipette; cellular morphology, while better than with the previous method, is not as good as a fresh specimen; appropriate precautions applicable to handling of potentially infective biological specimens need to be observed, particularly when cutting off the tips of the pipettes. Standardisation or quantification of the crystal load in each sample would help laboratories monitor improvement in performance over time.

Previous studies have clearly shown the need for QA programmes of synovial fluid analysis. The new method of synovial fluid preparation described allows many specimens to be prepared from a single sample, and for the specimens to be successfully transported over large distances without major deterioration in quality, thus increasing the likelihood that synovial fluid analysis will join most other laboratory investigations in routine QA programmes.

The authors thank the staff of RCPA Quality Assurance Programs Pty Ltd, especially Margot de Borde: Brian Nightingale, Ian Gould and staff, Royal Prince Alfred \& Rachel Forster Hospitals for participating in this study. This study was funded by the Department of Rheumatology, Royal Prince Alfred and Rachel Forster Hospitals. Distribution costs were donated by RCPA Quality Assurance Programs Pty Ltd.

1 Schumacher HR, Sieck MS, Rothfuss S, Clayburne GM, Baumgarten DF, Mochan BS, et al. Reproducibility of synovial fluid analyses. A study among four laboratories. synovial fluid analyses. A study

2 Hasselbacher P. Variation in synovial fluid analysis by hospital laboratories. Arthritis Rheum 1987;30:637-42.

3 Von Essen R, Holtta AMH. Quality control of the laboratory diagnosis of gout by synovial fluid microscopy. Scand J Rheumatol 1990;19:232-4.

4 McGill NW, York HF. Reproducibility of synovial fluid examination for crystals. Aust NZ J Med 1991;21:710-3.

5 Schumacher HR, Sieck M, Clayburne G. Development and evaluation of a method for preservation of synovial fluid wet preparations for quality control testing of crystal idenwet preparations for quality control testing

6 McGill NW, Swan A, Dieppe PA. Survival of calcium pyrophosphate dihydrate crystals in stored synovial fluids. Ann Rheum Dis 1991;50:939-41. 